\title{
Spatial Structure of Star Clusters by the 2MASS Database
}

\author{
J. W. Chen and W. P. Chen \\ Institute of Astronomy, National Central University, Chung-Li 32054, \\ Taiwan, Email: m899008@astro.ncu.edu.tw
}

\begin{abstract}
We present some results of a pilot program to study star clusters with the Two-Micron All-Sky Survey (2MASS) observations. While 2MASS cannot resolve the cores or detect much of the main sequence of globular clusters, the homogeneity and large angular coverages make the database suitable to study young star clusters. We show that member stars are centrally concentrated in open clusters, with a density distribution markedly shallower than that for globular clusters. In NGC2506 (age $3 \mathrm{Gyr}$ ) giant stars appear to occupy a smaller region than main sequence stars-a natural consequence of mass segregation.
\end{abstract}

\section{Spatial Distribution in a Star Cluster}

The stellar distribution in a young star cluster is dictated by the conditions in the molecular cloud from which the cluster was formed and, as the cluster evolves, the dynamical interaction among member stars, and the disruption by Galactic tidal force or differential rotation. An investigation of spatial structure in young clusters may shed light on the role each effect plays during the cluster evolution. The youngest clusters may bear more imprints of star formation, or even the molecular cloud structure, than the subsequent dynamical history.

Stars in a globular cluster are known to concentrate progressively toward the center. The King model-successfully applied to globular clusters, some open clusters and dwarf elliptical galaxies (King 1962) - is understood as a combination of an isothermal sphere (i.e., dynamically relaxed system) in the inner part of a cluster, and tidal truncation by the Milky Way in the outer part.

\section{Star Clusters in the 2MASS Database}

Fig. 1 shows an example of the (projected) number density distribution estimated by counting numbers of stars detected by 2MASS per square arcminute in concentric annuli-of the globular cluster M55. The radius of a cluster, $\mathbf{R}_{\mathrm{s}}$ is defined to be where the number density drops to 3- $\sigma$ of the background. The background is found to be rather uniform out to large angular extents, demonstrating the advantages of the wide sky coverage with the 2MASS data. A large background coverage is essential as distinction between cluster members and background stars is addressed in a statistical sense. While the central part of M55 is too crowded to resolve, the outer part is well fitted by the King model (Fig. 1). With its sensitivity, the 2MASS in general would not detect much of the main sequence in globular clusters. 
In each of the two open clusters presented here, NGC2506 (1.9 Gyr, Twarog et al 1999) and IC348 ( $\sim 6 \mathrm{Myr}$, Lada \& Lada 1995), the density profile is shallower than that for globular clusters. Fig 2 compares the cumulative density distributions of these two open clusters and the globular clusters, M55 and M13, within their respective $R_{s}$. In NGC2506, the bright giant stars (presumably more massive and evolved) seem to concentrate more toward the center, lending evidence of mass segregation in this open cluster.

The 2MASS database is proven a powerful tool for our application, and we are in the process of extending our pilot study to the analysis of a sample of young star clusters to determine their spatial structure. We plan to address the mass segregation history, and for the youngest clusters, the comparison with the density profiles or mass distribution in molecular cloud fragments.
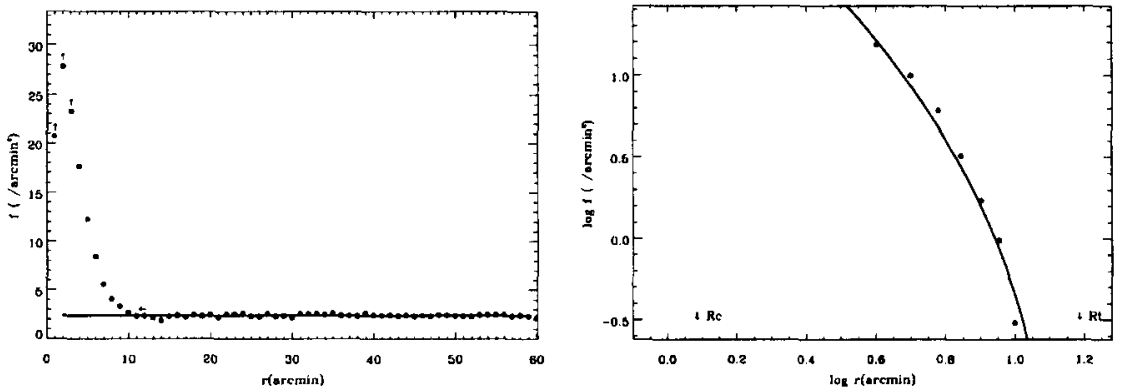

Figure 1. Linear (left) and logarithmic (right) star density profile.
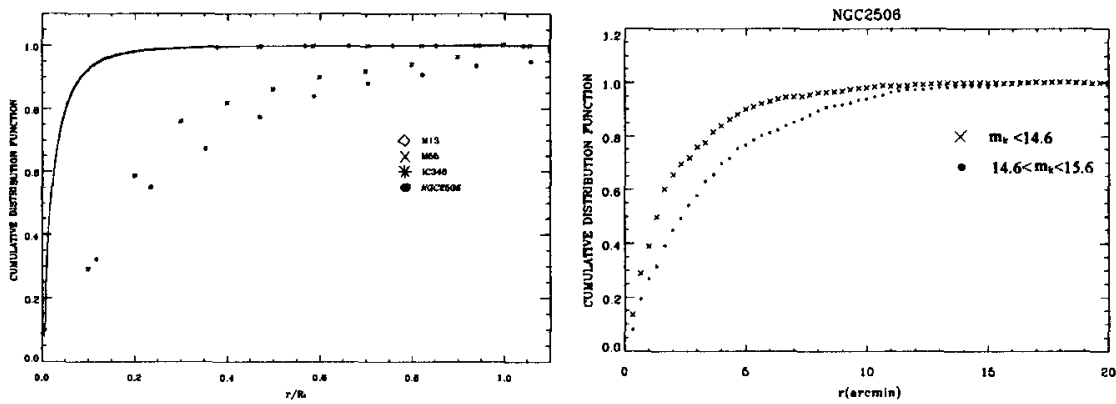

Figure 2. Cumulative density profiles (left) of globular and open clusters, and (right) of NGC2506.

\section{References}

King, I. 1962, AJ, 67, 471

Twarog, B. A., Anthony-Twarog, B. J. \& Bricker, A. R. 1999, AJ, 117, 1816

Lada, E. A. \& Lada, C. J. 1995, AJ, 109, 1682 